\title{
Antena de parche semicircular con elemento parásito para CanSat
}

\author{
Cruz Ángel Figueroa Torres ${ }^{1}$, José Luis Medina Monroy² ${ }^{2}$ Andrés Calvillo Téllez ${ }^{3}$ \\ ${ }^{1}$ Universidad Autónoma de Baja California, Ensenada, B. C., México \\ cruz.figueroa@uabc.edu.mx \\ ${ }^{2}$ Centro de Investigación Científica y de Educación Superior de Ensenada, Ensenada, B. C., \\ México \\ jmedina@cicese.mx \\ ${ }^{3}$ Instituto Politécnico Nacional, CITEDI, Tijuana, B. C., México \\ calvillo@citedi.mx
}

Resumen. En este trabajo se presenta el diseño de una antena de parche de estructura geométrica semicircular y con un elemento parásito, para su utilización en la radiocomunicación de satélites educativos de la categoría lata (CanSat). La antena desarrollada presentó un peso mínimo de 13.2 gramos, realizada físicamente en un material que cubre los requerimientos de tipo electromagnético, impresa en un substrato FR-4, con dimensiones máximas de $54 \mathrm{~mm}$ de diámetro, a una frecuencia resonante de $2.4 \mathrm{GHz}$. La antena tiene un peso, aproximadamente del $2 \%$ del peso total del nano satélite $(0.355 \mathrm{Kg})$. Las pérdidas por retorno $\mathrm{S} 11$ logradas son menores a $-10 \mathrm{~dB}$. Los resultandos obtenidos, como la ganancia, patrón de radiación, ancho de banda, y principalmente sus dimensiones y peso, exhiben a la antena propuesta como una gran opción para ser empleada en nanosatélites.

Palabras clave: CanSat, antena de parche parasito, antena semicircular.

\section{Semicircular Parasitic Patch Antenna for CanSat}

\begin{abstract}
This paper presents the design of a patch antenna with a semicircular geometric structure and a parasitic element, for its use in the radio communication of educational satellites of the can category (CanSat). The developed antenna presented a minimum weight of 13.2 grams, physically made of a material that covers the requirements of the electromagnetic type, printed on a substrate FR-4, with maximum dimensions of $54 \mathrm{~mm}$ in diameter, at a resonant frequency of $2.4 \mathrm{GHz}$. The antenna has a weight, approximately $2 \%$ of the total weight of the nano satellite $(0.355 \mathrm{Kg})$. The return losses $\mathrm{S} 11$ achieved are less than $-10 \mathrm{~dB}$. The obtained results, such as gain, radiation pattern, bandwidth, and mainly its dimensions and weight, show the proposed antenna as a great option to be used in nanosatellites.
\end{abstract}

Keywords: CanSat, parasitic patch antenna, semicircular antenna. 


\section{Introducción}

Un CanSat es un prototipo de satélite destinado a la educación en la formación de recursos humanos en matemáticas, ciencia y tecnología. El diseño del CanSat debe ajustarse a las dimensiones y limitaciones de masa, sus componentes y subsistemas deben ser más pequeños en comparación con los satélites convencionales. Actualmente los satélites educativos tienen un papel importante para el desarrollo de tecnología en las agencias espaciales, industria e instituciones educativas.

Se puede conceptualizar a un CanSat como un prototipo educativo a escala de un satélite mayor. Una misión típica consta de: captura de video, toma de fotografías aéreas, y mediciones de las condiciones climáticas y atmosféricas. El CanSat puede realizar las funciones de colectar información de la carga útil y transmitirla a una estación terrena. Siendo la antena el elemento esencial para la transmisión formando parte del módulo de comunicaciones que integran el satélite educativo. Este prototipo de CanSat, ha logrado captar la atención de la comunidad académica como una forma de materializar teorías en aplicaciones espaciales. De tal forma que el desarrollo tecnológico forme capital humano en esta área, altamente valorado [1].

$\mathrm{Su}$ reducida dimensión permite que se construyan rápidamente, mientras que el peso determina el bajo costo para el lanzamiento. Los elementos básicos que lo constituyen son: fuente de alimentación, subsistema de computadora de abordo, subsistema de adquisición de variables físicas y el subsistema de comunicaciones, constituido por el transpondedor y la antena.

Una forma económica de hacer investigación y desarrollo tecnológico en el área de comunicaciones en especial antenas, es utilizar tarjetas de circuito impreso para señales de radiofrecuencia de material FR-4 sin embargo presenta un nivel de pérdidas significativo cuando opera señales electromagnéticas de alta frecuencia, con respecto al material de alto valor económico, que posee características eléctricas superiores como lo es el de teflón o politetrafluoroetileno (PTFE), esto implica que se espera que el diseño físico presente variaciones de funcionamiento respecto al modelo del diseño [2].

Entre las principales aplicaciones están el realizar investigación espacial, medio ambiente del espacio, investigación atmosférica, astronomía, ciencias planetarias, monitoreo del medio ambiente terrestre, entre otras [3-9].

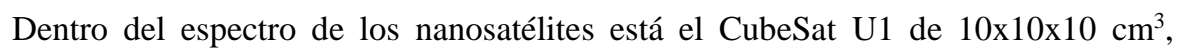
mientras que el CubeSat de $3 \mathrm{U}$ tiene un volumen de 30x10x10 $\mathrm{cm}^{3}$. El peso total de este nanosatélite es de $1 \mathrm{Kg}$, aunque pueden existir algunas variaciones [10-14].

Otro tipo de nano satélite es el CanSat, propuesto por el profesor Robert Twiggs de la Universidad de Stanford en 1999, en una lata de refresco (355ml) [1]. El desarrollo lo promueve la Agencia Espacial Mexicana (AEM), para capacitación en el sector Aeroespacial y con el deseo de que los mejores equipos de trabajo mexicanos puedan participar con sus desarrollos a nivel internacional [1].

La agencia encargada de la regulación de la frecuencia de 2.4 a $2.5 \mathrm{MHz}$ para satélites de aficionados es la Unión Internacional de Telecomunicaciones (ITU) [11]. 


\section{Desarrollo}

Existen escasos diseños para aplicaciones en nanosatélites, en especial en la categoría de los CanSat. En este trabajo se presenta una antena que puede ser colocada en la cara superior o inferior del CanSat. Las especificaciones de diseño para la antena propuesta son: frecuencia de operación en $2.45 \mathrm{GHz}$ y con un ancho de banda de $100 \mathrm{MHz}$.

En la figura 1 se puede observar la estructura propuesta en este artículo. Se sabe que la frecuencia de resonancia de un elemento circular está determinada directamente por su diámetro y el punto de alimentación. Para calcular la frecuencia de resonancia del elemento radiador se emplearon las ecuaciones propuestas en [15].

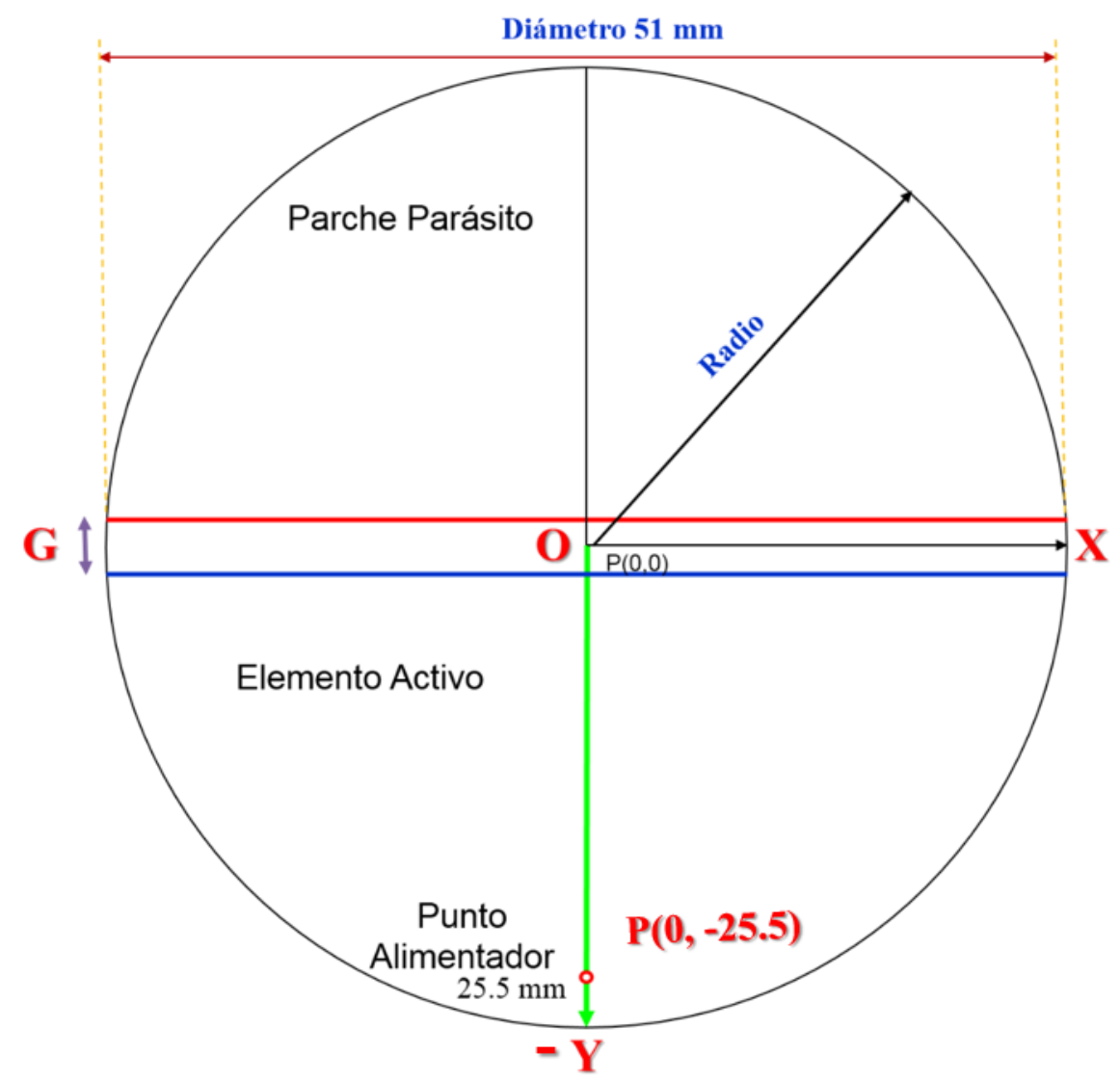

Fig. 1. Antena semicircular de parche con elemento parásito para CanSat.

El parche circular tiene un diámetro $\mathrm{D}=51 \mathrm{~mm}$. El puerto de alimentación está centrado en el parche, es decir en $\mathrm{Py}=25.5 \mathrm{~mm}$. El punto de alimentación es un factor 
crítico para establecer la frecuencia de operación de la antena, por esta razón, estos parámetros también son optimizados mediante la técnica de algoritmos genéticos, para determinar la ubicación del punto de alimentación dónde las pérdidas por retorno (parámetro S11) sean adecuadas, es decir que cumplan con la función objetivo. Cabe señalar que el parámetro S11 determina la impedancia de entrada de un dispositivo. El primer paso es definir la función objetivo centrado en las pérdidas por retorno (S11), las cuales deben ser menores a $-10 \mathrm{~dB}$ en la banda de operación requerida. La Función objetivo queda expresada de la siguiente manera:

$$
\text { Función objetivo }=\operatorname{Min}(S 11)_{2.45 \mathrm{GHz}}
$$

La tabla 1 presenta los rangos las variables a optimizar y el espacio de decisión.

El valor resultante de la abertura $G$ es de $0.5 \mathrm{~mm}$, el cual fue optimizado para sintonizar la frecuencia deseada, mediante el programa de cómputo de análisis electromagnético CST.

Tabla 1. Distancias de la separación de la antena y el elemento parasitó y del punto central de la antena sobre el eje Y.

\begin{tabular}{cc}
\hline Parámetro & Espacio de decisión $(\mathbf{m m})$ \\
\hline$G$ & $0.1-1$ \\
Py & $-6--25.5$ \\
\hline
\end{tabular}

En la figura 2 se puede apreciar el comportamiento de la impedancia de entrada de la antena para diferentes ubicaciones del punto de alimentación con respecto al eje "y". Se sabe que la ubicación del punto de alimentación de una antena contribuye a determinar su frecuencia de resonancia y a mejorar su acoplamiento (S11<-10 dB). Por lo cual, es necesario realizar un análisis paramétrico para identificar cuál es la ubicación optima del puerto de alimentación, que garantice el mayor ancho de banda y el mejor acoplamiento en la banda de interés.

La figura 2 muestra algunos de los resultados del análisis paramétrico, los cuales se realizaron con ayuda del software de análisis electromagnético CST. Este análisis fue realizado desde $\mathrm{Py}=0$ hasta $-25.5 \mathrm{~mm}$ con pasos de $0.5 \mathrm{~mm}$. Para ello se seleccionaron solo algunas curvas, con el objetivo de mostrar cómo influye la ubicación del puerto de alimentación en la frecuencia de resonancia. Con $\mathrm{Py}=-25$ el ancho de banda se redujo considerablemente, por lo cual se optó por seleccionar $\mathrm{Py}=-25.5$. Con ello se puede observar que cuando el puerto de alimentación está localizado en Py $=-25.5 \mathrm{~mm}$ se cubre la banda de $2.43-2.54 \mathrm{GHz}$ para un S11<-10 dB. Esta banda cumple con las especificaciones de diseño del CanSat.

Además, en base a nuestra experiencia en diseño de antenas, es conocido que cuando la antena sea colocada en la estructura del CanSat la frecuencia de resonancia tenderá a desplazarse hacia abajo un par de $\mathrm{MHz}$, debido a que la estructura de los bordes del satélite hará ver que la geometría de la antena aumentó unos $\mathrm{mm}$, con lo cual la frecuencia de resonancia tenderá a reducirse. 


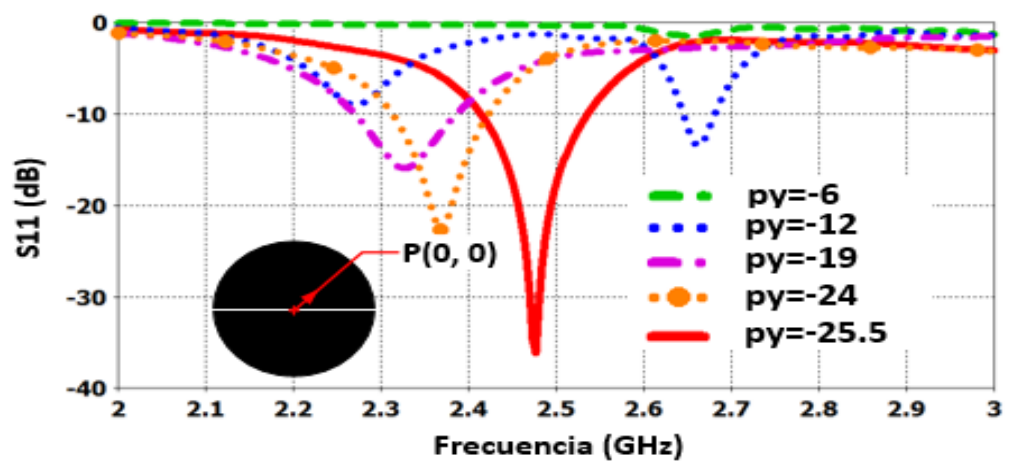

Fig. 2. Análisis del punto de alimentación.

\section{Resultados}

La antena fue construida en un substrato FR-4, el cual es económico con respecto al PTFE y fácil de conseguir. La antena está impresa sobre un substrato con diámetro de $54 \mathrm{~mm}$ y un espesor de $3.1556 \mathrm{~mm}$, tal como se observa en la figura 3 .

Una ventaja importante de este diseño es el peso, el cual es $13.5 \mathrm{~g}$, lo cual representa menos del $2 \%$ del peso total de un nanosatélite.
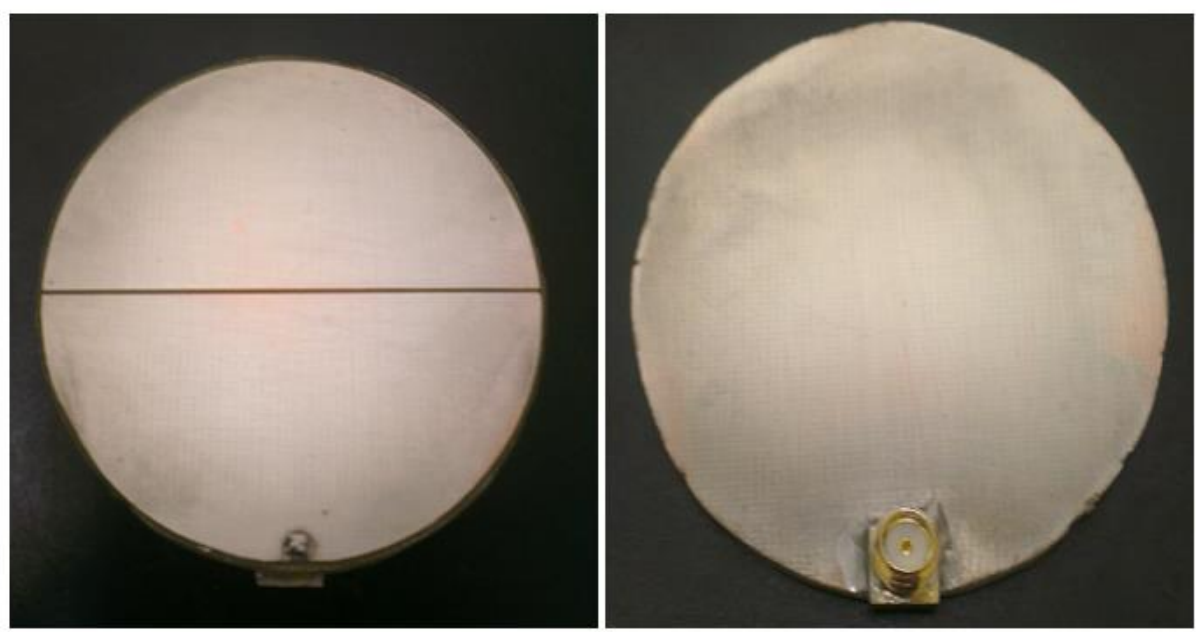

Fig. 3. Antena fabricada: a) vista superior, b) vista inferior.

La antena se diseñó y analizó electromagnéticamente utilizando el Software Computer Simulation Technology (CST). En la figura 4 se realiza una comparación entre los resultados simulados y medidos del parámetro S11, el cual nos determina la frecuencia de resonancia y el ancho de banda de la antena. El ancho de banda se obtiene a partir de $\mathrm{S} 11 \leq-10 \mathrm{~dB}$. En la figura se puede observar que el rango de frecuencia de 
operación medido es de $2.43 \mathrm{GHz}-2.54 \mathrm{GHz}$. Mientras que el simulado es de 2.41 $\mathrm{GHz}-2.54 \mathrm{GHz}$ ambos para un S11<-10dB. Se hace notar que el rango de frecuencia de operación fue medido sin considerar el efecto del satélite. Anteriormente mencionamos que este efecto provocará que la frecuencia de resonancia se desplace hacia abajo un par de MHz. Sería interesante realizar la medición de la antena una vez que el satélite cuente con todas sus etapas, para ver en qué medida se reduce la frecuencia de resonancia y con esto se puede advertir a los diseñadores de antenas para que consideren esta cantidad en sus diseños. Se puede notar una pequeña variación entre el resultado simulado y el medido, esta se debe a pequeñas variaciones en las dimensiones de la estructura ocasionadas en el proceso de fabricación.

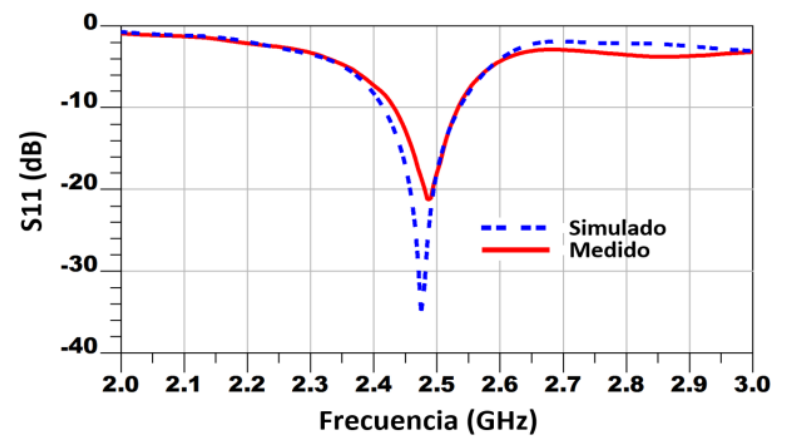

Fig. 4. Parámetro S11 antena.

La ganancia de la antena tiene un papel muy importante en el comportamiento de la señal emitida o recibida. Por lo tanto, es necesario conocer la ganancia de la antena con exactitud, para poder determinar el nivel recibido por un receptor o el nivel emitido por un transmisor.

La ganancia de una antena depende de: el número de elementos que la forman, las dimensiones del elemento radiador, espesor del substrato, entre otras. Y se asocia con su directividad.

Una antena con ganancia alta será más directiva, permitiendo concentrar la energía en una dirección en específico y disminuir el nivel de sus lóbulos laterales. En aplicaciones móviles se requieren antenas con gran cobertura, es decir, con baja directividad.

Existen varios métodos y configuraciones que se utilizan para medir la ganancia, y que dependen de la frecuencia de operación [16-17].

A frecuencias superiores a $1 \mathrm{GHz}$, usualmente se utiliza el espacio libre para medir la ganancia, puesto que a frecuencias menores es difícil simular las condiciones de espacio libre debido a las longitudes de onda grandes, razón por la cual se mide bajo condiciones con reflexión a tierra [16].

En la figura 5 se puede observar la ganancia de la antena para el rango de frecuencias de $2.4 \mathrm{GHz}$ a $3 \mathrm{GHz}$.

La medición de ganancia se llevó a cabo utilizando un analizador de espectros, un generador de señales de microondas y además una antena patrón o de referencia. 
El procedimiento de medición es el siguiente: se realiza un barrido en frecuencia y se efectúa la medición de potencia en cada frecuencia.

La ganancia para la frecuencia de $2.45 \mathrm{GHz}$ es de $3 \mathrm{dBi}$.

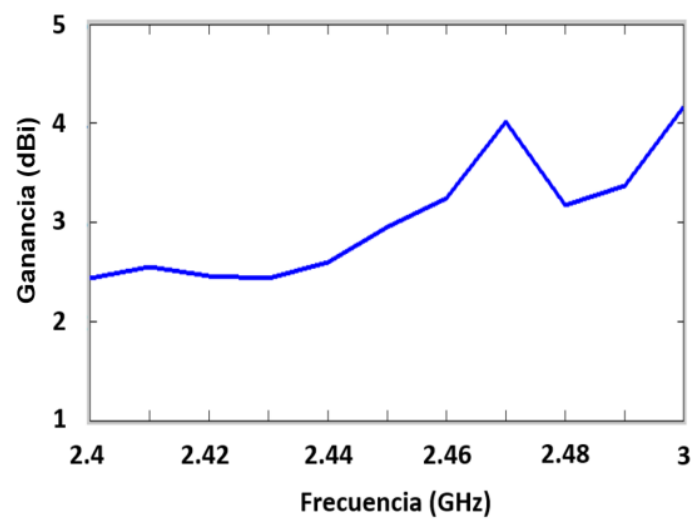

Fig. 5. Ganancia de la antena.

En la figura 6 se muestra la antena colocada sobre una lata de refresco, la cual simula un dispositivo CanSat, esto con el fin de demostrar que la antena propuesta en este trabajo de investigación cumple con los requerimientos de tamaño para ser utilizada en un nano satélite.

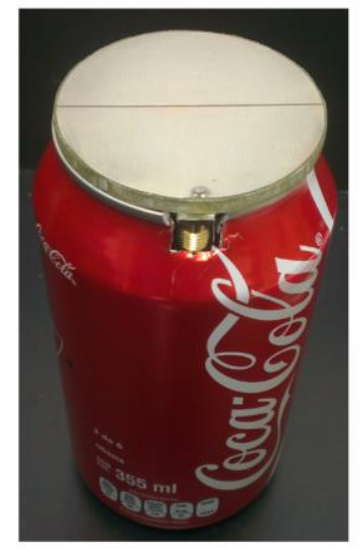

Fig. 6. Satélite en lata con antena semicircular de parche con elemento parásito.

El patrón de radiación es una representación gráfica de las propiedades de radiación de la antena en función de las coordenadas espaciales. En la mayoría de los casos, el patrón de radiación es determinado para la región de campo lejano. En la figura 7 se puede apreciar el patrón de radiación para la frecuencia de $2.4 \mathrm{GHz}$. El ancho del haz de mediana potencia (HPBW) es de $98.2^{\circ}$ y la magnitud del lóbulo principal es de 5.4 
Cruz Ángel Figueroa Torres, José Luis Medina Monroy, Andrés Calvillo Téllez

dBi. El nivel de los lóbulos laterales es de -10.4 dB. Debido a que la antena tiene plano de tierra en la parte posterior del substrato, el nivel de los lóbulos laterales es bajo.

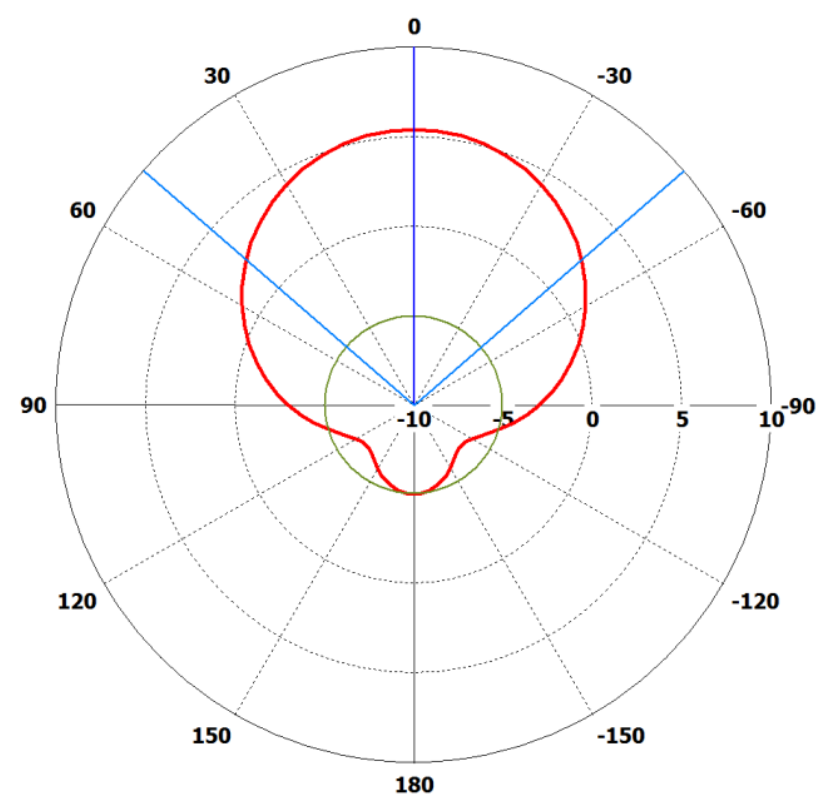

Fig. 7. Patrón de radiación a $2.4 \mathrm{GHz}$.

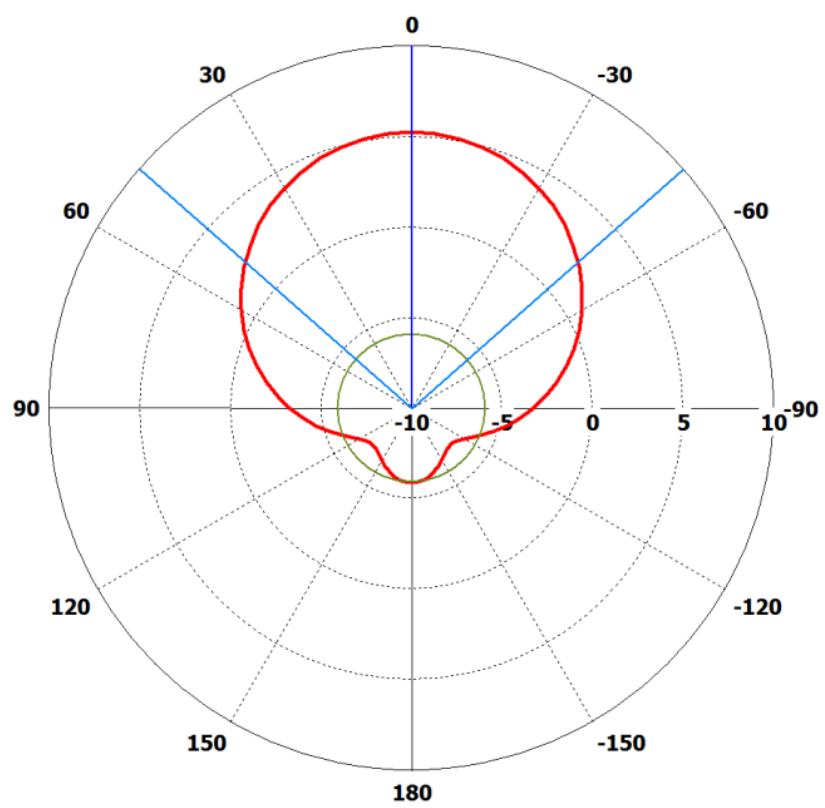

Fig. 8. Patrón de radiación a $2.45 \mathrm{GHz}$. 


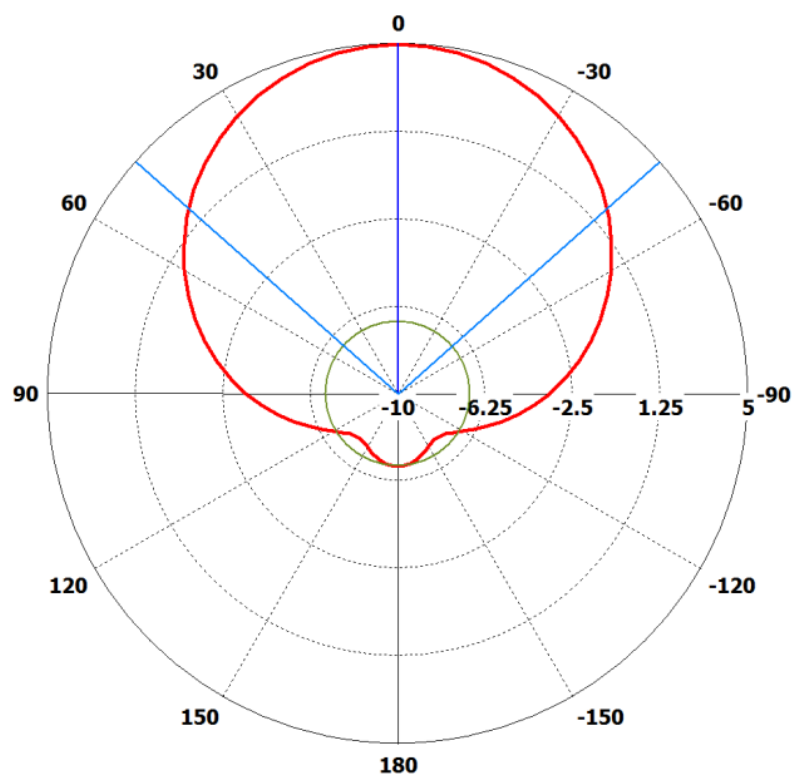

Fig. 9. Patrón de radiación a $2.5 \mathrm{GHz}$.

Para comprender adecuadamente que la antena sólo radia en una sola dirección, es decir sobre el parche circular, se realiza el análisis electromagnético del patrón de radiación en 3D para la frecuencia de $2.45 \mathrm{GHz}$, el cual se muestra en la figura 10.

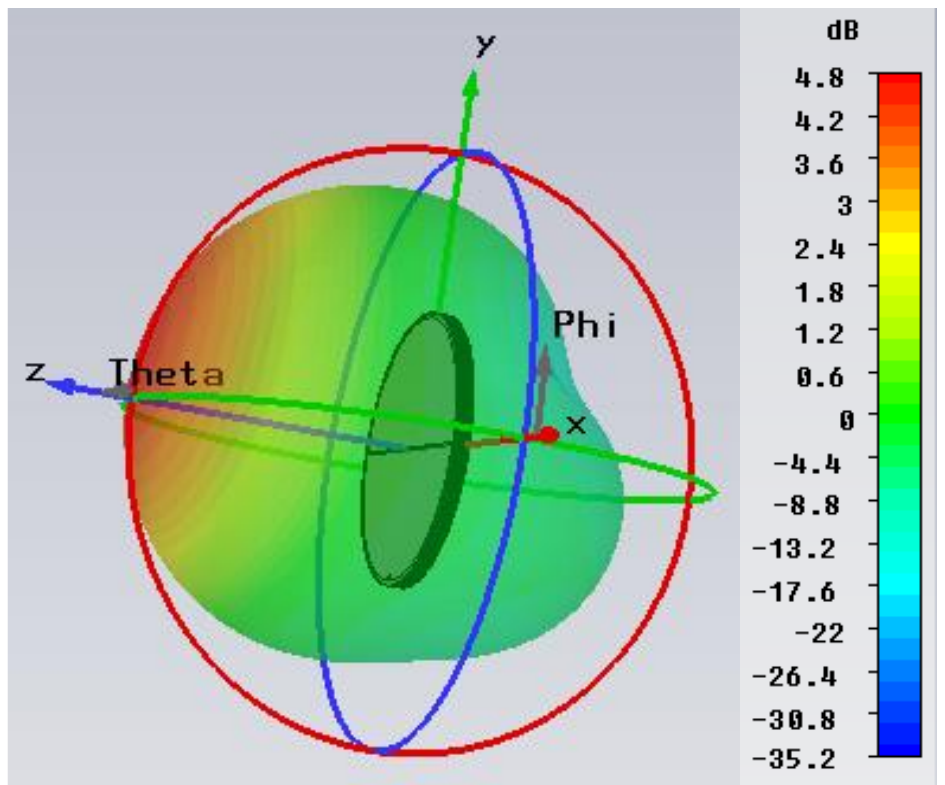

Fig. 10. Patrón de radiación 3D a 2.45 GHz. 
En la figura 11 se puede observar que la máxima eficiencia obtenida en el diseño propuesto es de $80 \%$, siendo esta para la frecuencia de $2.35 \mathrm{GHz}$. Mientras que la eficiencia mínima obtenida es de $15 \%$ para la frecuencia de $2.65 \mathrm{GHz}$. Cabe resaltar que la antena tiene buena eficiencia (> $60 \%$ ) en la frecuencia de operación del CanSat, es decir en $2.4 \mathrm{GHz}$. La baja eficiencia en algunas frecuencias es debido a que la antena no es diseñada para operar en tales frecuencias.

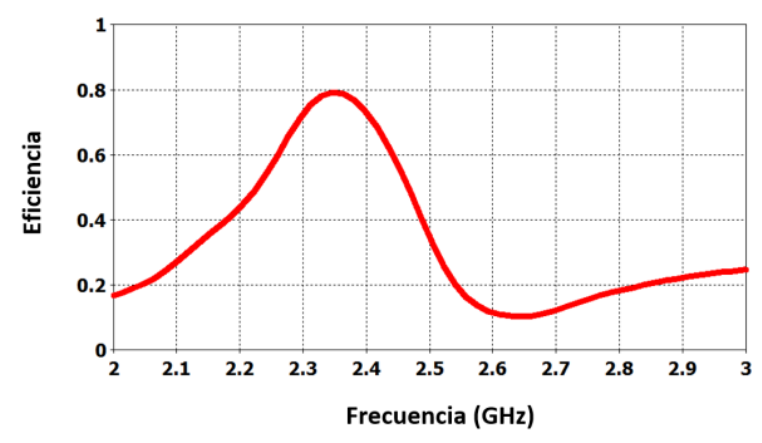

Fig. 11. Eficiencia de la antena.

\section{Discusión}

La antena mostró parámetros que satisfacen en ganancia, directividad, impedancia de entrada y el patrón de radiación con niveles de lóbulos laterales. Existen algunos diseños de antenas orientadas a nanosatélites, en [13], muestra un diseño de antena rómbica, cuyo porcentaje de ancho de banda es de $7.5 \%$ y sus dimensiones son $10 \times 10 \mathrm{~cm} 2$, con un peso de $157 \mathrm{~g}$, el cual representa el $15.7 \%$ del peso del CubeSat, por lo cual la antena limita en peso la construcción de las etapas de detección, amplificación y procesamiento de la señal. Con el objetivo de aumentar la ganancia y por consecuencia la directividad, en el trabajo presentado en [6], se utiliza un reflector inflable para la antena. Dicha estructura proporciona una ganancia de $9 \mathrm{dBi}$, sin embargo, el reflector requiere un tanque presurizado, por ello, la antena tiene un periodo de vida corto. En este artículo se presentó una antena que se ha diseñado y construido, la antena es una estructura original, la cual cumple con los requerimientos fundamentales para la integración en nanosatélites, como lo son: el peso y las dimensiones de la antena. En este trabajo se incluyen mediciones de: la impedancia de la antena, ganancia y patrón de radiación.

\section{Conclusiones}

En este artículo se presentan los resultados del diseño, optimización y construcción de una antena que puede emplearse en nanosatélites. La ventaja de esta antena comparada con otras es: la fabricación en un substrato de bajo costo, pero sobretodo el tamaño, dado que dicha estructura no aumenta el área ocupada por el sistema. 
El peso de la antena es una de las ventajas más importantes de este diseño, el cual es de tan solo 13.5 g. Además, la antena presenta un buen comportamiento en ancho de banda, el cual es de $100 \mathrm{MHz}$. Por otra parte, la ganancia obtenida es de 3dBi para la frecuencia de $2.45 \mathrm{GHz}$.

El patrón de radiación mostrado por la antena es omnidireccional, lo cual es muy útil, debido a que se tiene un área de cobertura amplia.

Contrario a lo que normalmente se puede encontrar en la literatura si bien en el diseño de la antena propuesta se emplean metodologías y modelos ya conocidos; la antena de parche semicircular con elemento parásito para CanSat es una novedad, debido a que después de indagar en el estado del arte, no se encontró una topología semejante en aplicaciones en CanSat. Esto la hace una aportación de interés para los nuevos prototipos de satélites pequeños. Actualmente se continúan usando la mayoría de desarrolladores de este tipo de satélites antenas dipolo, las cuales ocupan demasiado espacio, incrementan el peso y sacan de balance al CanSat.

\section{Referencias}

1. Navarro Lieja, D.: Integración de un satélite CanSat y propuesta de sus medios de lanzamiento para capacitar recursos humanos a nivel universitario. Tesis de Maestría, UNAM, (2015)

2. Coonrod, J.: Bending and Forming High Frequency Printed Circuits. IPC Printed Circuits Expo, APEX and the Designers Summit (2007)

3. Nascetti, A., Pittella, E., Teofilatto, P., Pisa, S.: High-Gain S-band patch antenna system for Earth-Observation CubeSat Satellites. IEEE Antennas and Wireless Propagation Letters, Vol. 14, pp. 434-437 (2015)

4. Swartwout, M.: The first one hundred CubeSats: A statistical look. Journal of JoSS Small Satellite, Vol. 2, pp. 213-233 (2014)

5. Sandau, R.: Status and trends of small satellite missions for earth observation. Acta Astronautica, Vol. 66, pp. 1-12 (2010)

6. Mahmoud, A., Elazhary, T., Zaki, A.: Remote sensing CubeSat. In: Proceedings SPIE, Vol. 7826, pp. 78262I-78262I-8 (2010)

7. Babuscia, A., et al.: Commcube 1 and 2: A CubeSat series of missions to enhance communication capabilities for cubesat. In: Proceedings IEEE Aerospace conference, pp. 119 (2013)

8. Nugent, R., Munakata, R., Chin, A., Coelho, R., Puig-Suari, J.: The CubeSat: The picosatellite standard for research and education. Aerospatiale Eng, Vol. 805, pp. 756-5087 (2008)

9. Toorian, A., Diaz, K., Lee, S.: The CubeSat approach to space access. In: Proceedings IEEE Aerospace conference, pp. 1-14 (2008)

10. Heidt, H., Puig-Suari, J., Moore, A. S., Nakasuka, S., Twiggs, R. J.: CubeSat: A new generation of picosatellite for education and industry low-cost space experimentation. In: Proceedings $14^{\text {th }}$ annual USU Small satellite conference, SSC00-V-5 (2000)

11. Handbook of frequency allocations and spectrum protection for Scientific uses. Committee on radio frequencies council (2007)

12. CubeSat program and community official. Available at: http://cubesat.atl.calpoly.edu/

13. Rudge, A. W., Milne, K., Olver, A. D., Knight, P.: The handbook of antenna design. London, Peter Peregrinus Ltd. (1986) 
Cruz Ángel Figueroa Torres, José Luis Medina Monroy, Andrés Calvillo Téllez.

14. Pedroza, J. S. , Peña, S., Martínez-Zúñiga, F.: A $2.4 \mathrm{GHz}$ cross rhombic antenna for a cube satellite application. International Journal of Antennas and propagation, Vol. 2014 (2014)

15. Babuscia, A., Van de Loo, M., Wei, Q. J., Pan, S., Mohan, S., Seager, S.: Inflatable antenna for CubeSat: fabrication, deployment and results of experimental test. In: Proc. IEEE Aerospace conference, pp. 1-12 (2014)

16. Verma, K., Nasimuddin, N.: Simple expressions for the directivity of a circular microstrip antenna. IEEE antennas and propagation magazine, Vol. 44, pp. 91-95 (2002)

17. Balanis Constantine, A.: Antenna Theory: Analysis and design. John Wiley and Sons, $2^{\text {nd }}$ ed., New York, p. 941 (1997) 